\title{
Conformal mappings of bounded multiply connected regions onto circular and parallel slits regions and their inverses using a GUI
}

\author{
Ali W.K. Sangawi ${ }^{a, b}$, Ali H.M. Murid ${ }^{c, d, *}$, Khiy Wei Lee ${ }^{c, d}$ \\ a Department of Computer, College of Basic Education, Charmo University, 46023 Chamchamal, \\ Sulaimani, Kurdistan, Iraq \\ b Development Centre for Research and Training-University of Human Development, 46001 Sulaimani, \\ Kurdistan, Iraq \\ c UTM Centre for Industrial and Applied Mathematics (UTM-CIAM), Universiti Teknologi Malaysia, \\ 81310 UTM Johor Bahru, Johor, Malaysia \\ d Department of Mathematical Sciences, Faculty of Science, Universiti Teknologi Malaysia, \\ 81310 UTM Johor Bahru, Johor, Malaysia
}

*Corresponding author, e-mail: alihassan@utm.my

Received 22 Aug 2014

Accepted 21 May 2017

\begin{abstract}
We present a boundary integral equation method for the numerical conformal mappings and their inverses of bounded multiply connected regions onto circular and parallel slits regions. The method is based on two uniquely solvable boundary integral equations with Neumann-type and generalized Neumann kernels. These boundary integral equations are constructed from a boundary relationship satisfied by a function analytic on a multiply connected region. A method to calculate the inverse mapping functions from circular and parallel slits regions onto the original region is presented. Some numerical examples and numerical results with the graphical user interface are presented to illustrate the efficiency of the presented method.
\end{abstract}

KEYWORDS: numerical conformal mapping, boundary integral equations, Neumann-type kernel, generalized Neumann kernel

MSC2010: 30Cxx 65Exx 65Dxx 65Rxx 51Mxx 68Qxx

\section{INTRODUCTION}

There are several types of canonical slit regions of conformal mapping ${ }^{1,2}$. Reformulations of conformal mappings from bounded and unbounded multiply connected regions onto the five canonical slit regions as Riemann-Hilbert $(\mathrm{RH})$ problems are discussed in Refs. 3-5. An integral equation with the generalized Neumann kernel is then used to solve the $\mathrm{RH}$ problem as developed in Ref. 6. Amano ${ }^{7}$ and DeLillo et $\mathrm{al}^{8}$ have constructed charge simulation method and least squares method, respectively, for approximate conformal mapping onto circular and radial slits regions.

A different integral equation approach for conformal mapping of bounded multiply connected regions onto canonical slits regions have been discussed in Refs. 9,10. Two papers are concerned with disc ${ }^{11}$ and annulus ${ }^{12}$ with slits which improve the work of Murid and $\mathrm{Hu}^{13,14}$.
This paper presents a new integral equation method with the adjoint generalized Neumann and Neumann-type kernel for conformal mappings and their inverses of bounded multiply connected regions onto a circular slits region and parallel slits region which extends the work presented in Refs. 9,10. Unlike the methods presented in Refs. 9, 10 that require solving three integral equations, this paper involves two integral equations only.

We derive integral equations related to $f^{\prime}$ for circular slits region $\Omega_{1}$ and parallel slits region $\Omega_{2}$, where $f$ is a conformal mapping of bounded multiply connected regions onto $\Omega_{1}$ and $\Omega_{2}$. Methods to compute the interior and inverse mapping functions for $\Omega_{1}$ and $\Omega_{2}$ are also given. We give some examples to illustrate our boundary integral equation method. We also give some descriptions about graphical user interface for circular and parallel slits maps. 

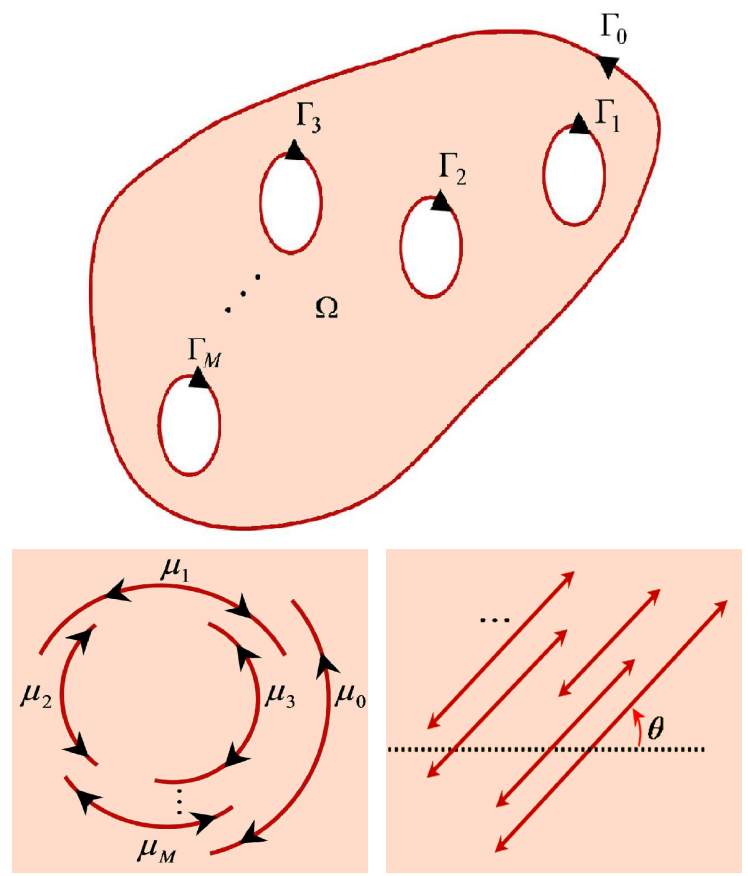

Fig. 1 Mapping of a bounded multiply connected region $\Omega$ onto the canonical slits regions $\Omega_{1}$ and $\Omega_{2}$.

\section{NOTATION AND AUXILIARY MATERIALS}

Let $\Omega$ be a bounded multiply connected region of connectivity $M+1$ with $0 \in \Omega$. The boundary $\Gamma$ consists of $M+1$ smooth Jordan curves $\Gamma_{j}, j=$ $0,1, \ldots, M$ (Fig. 1).

The curves $\Gamma_{j}$ are parameterized by $2 \pi$-periodic twice continuously differentiable complex functions $z_{j}(t)$ with non-vanishing first derivatives

$$
z_{j}^{\prime}(t)=\mathrm{d} z_{j}(t) / d t \neq 0,
$$

where $t \in J_{j}=[0,2 \pi]$ for each $j=0,1, \ldots, M$. The total parameter domain $J$ is the disjoint union of $M+1$ intervals $J_{0}, \ldots, J_{M}$. We define a parameterization $z(t)$ of the whole boundary $\Gamma$ on $J$ by

$$
z(t)=z_{j}(t) \quad\left(t \in J_{j}\right)
$$

Let $H^{*}$ be the space of all real Hölder continuous $2 \pi$-periodic functions $\omega(t)$ of the parameter $t$ on $J_{j}$ for $j=0,1, \ldots, M$, i.e.,

$$
\omega(t)=\omega_{j}(t) \quad\left(t \in J_{j}\right) .
$$

Suppose that $c(z), Q(z), H(z), T(z)$ are complex-valued functions defined on $\Gamma$ such that $c(z) \neq 0, H(z) \neq 0, Q(z) \neq 0, T(z(t))=z^{\prime}(t) /\left|z^{\prime}(t)\right|$ and $\overline{H(z)} /(T(z) Q(z))$ satisfies the Hölder condition on $\Gamma$. Then the interior relationship is defined as follows.

A complex-valued function $P(z)$ is said to satisfy the interior relationship if $P(z)$ is analytic in $\Omega$ and satisfies the non-homogeneous boundary relationship

$$
P(z)=c(z) \frac{\overline{T(z) Q(z)}}{\overline{G(z)}} \overline{P(z)}+\overline{H(z)} \quad(z \in \Gamma),
$$

where $G(z)$ is analytic in $\Omega$, Hölder continuous on $\Gamma$, and $G(z) \neq 0$ on $\Gamma$. The boundary relationship (2) also has the following equivalent form:

$$
G(z)=\overline{c(z)} T(z) Q(z) \frac{P(z)^{2}}{|P(z)|^{2}}+\frac{G(z) H(z)}{\overline{P(z)}} .
$$

The following theorem from Ref. 9 gives an integral equation for an analytic function satisfying the interior non-homogeneous boundary relationship (2) or (3).

Theorem 1 If the function $P(z)$ satisfies the interior non-homogeneous boundary relationship (2) or (3), then

$$
\begin{aligned}
& P(z)+f_{\Gamma} K(z, w) P(w)|\mathrm{d} w| \\
& +c(z) \overline{T(z) Q(z)} \overline{\sum_{a_{j} \in \Omega} \operatorname{Res}_{w=a_{j}} \frac{P(w)}{(w-z) G(w)}} \\
& =-\overline{T(z) Q(z) L_{\mathrm{R}}^{-}(z)} \quad(z \in \Gamma),
\end{aligned}
$$

where

$$
K(z, w)=\frac{1}{2 \pi \mathrm{i}}\left[\frac{c(z) \overline{T(z) Q(z)}}{c(w) \overline{(w-z) Q(w)}}-\frac{T(w)}{w-z}\right],
$$

when $z \neq w$ and

$$
\begin{aligned}
L_{\mathrm{R}}^{-}(z)= & \frac{-1}{2} \frac{H(z)}{Q(z) T(z)} \\
& +\frac{1}{2 \pi \mathrm{i}} \int_{\Gamma} \frac{\overline{c(z)} H(w)}{\overline{c(w)}(w-z) Q(w) T(w)} \mathrm{d} w .
\end{aligned}
$$

For every fixed points $z, w \in \Gamma$, the limit $K(z, w)$ exists as $w \rightarrow z$ and

$$
\lim _{w \rightarrow z} K(z, w)=-\frac{1}{2 \pi \mathrm{i}\left|z^{\prime}(t)\right|}\left[\overline{\left(\frac{q^{\prime}(t)}{q(t)}\right)}+\frac{\rho^{\prime}(t)}{\rho(t)}\right],
$$

where $q(t)=Q(z(t)), \rho(t)=c(z(t))$, and the minus sign in the superscript denotes the limit from the exterior. 
Let $\hat{A}(t)$ be a complex continuously differentiable $2 \pi$-periodic function for all $t \in J$. The generalized Neumann kernel formed with $\hat{A}$ is defined by $^{6,9}$

$$
\hat{N}(t, s)=\frac{1}{\pi} \operatorname{Im}\left(\frac{\hat{A}(t)}{\hat{A}(s)} \frac{z^{\prime}(s)}{z(s)-z(t)}\right) .
$$

This kernel is continuous with

$$
\hat{N}(t, t)=\frac{1}{\pi}\left(\frac{1}{2} \operatorname{Im} \frac{z^{\prime \prime}(t)}{z^{\prime}(t)}-\operatorname{Im} \frac{\hat{A}^{\prime}(t)}{\hat{A}(t)}\right) .
$$

The adjoint function to the function $\hat{A}$ is given by

$$
\tilde{A}(t)=\frac{z^{\prime}(t)}{\hat{A}(t)} .
$$

The generalized Neumann kernel $\tilde{N}(s, t)$ formed with $\tilde{A}$ is given by

$$
\tilde{N}(t, s)=\frac{1}{\pi} \operatorname{Im}\left(\frac{\tilde{A}(t)}{\tilde{A}(s)} \frac{z^{\prime}(s)}{z(s)-z(t)}\right) .
$$

Then

$$
\tilde{N}(s, t)=-\hat{N}^{*}(s, t),
$$

where $\hat{N}^{*}(s, t)=\hat{N}(t, s)$ is the adjoint kernel of the generalized Neumann kernel $\hat{N}(s, t)^{6}$. Define the Fredholm integral operator $\hat{\mathbf{N}}^{*}$ by

$$
\hat{\mathbf{N}}^{*} \psi(t)=\int_{J} \hat{N}^{*}(t, s) \psi(s) \mathrm{d} s \quad(t \in J) .
$$

In this paper $\hat{A}(t)=z(t)$. It is known that $\lambda=1$ is an eigenvalue of the kernel $\hat{N}$ with multiplicity 1 and $\lambda=-1$ is an eigenvalue of $\hat{N}$ with multiplicity $\hat{M}^{15}$. The eigenfunctions of $\hat{N}$ corresponding to the eigenvalue $\lambda=-1$ are $\left\{\chi^{[0]}, \chi^{[1]}, \ldots, \chi^{[M]}\right\}$, where ${ }^{15}$

$$
\chi^{[j]}(\xi)=\left\{\begin{array}{ll}
1 & \xi \in \Gamma_{j}, \\
0 & \text { otherwise, }
\end{array} \quad j=0,1, \ldots, M .\right.
$$

Define the space $\hat{S}$ by

$$
\hat{S}=\operatorname{span}\left\{\chi^{[0]}, \chi^{[1]}, \ldots, \chi^{[M]}\right\}
$$

and define an integral operator $\hat{\mathbf{J}}$ by ${ }^{9}$

$$
\hat{\mathbf{J}} v=\int_{J} \frac{1}{2 \pi} \sum_{j=0}^{M} \chi^{[j]}(s) \chi^{[j]}(t) v(s) \mathrm{d} s .
$$

The following theorem will be useful in the following sections for calculating the piecewise real function $h(t)$ in canonical slit representation ${ }^{9}$.
Theorem 2 Suppose the functions $\gamma \in H^{*}, h, \mu \in \hat{S}$ are such that

$$
\hat{A} \tilde{g}=\gamma+h+\mathrm{i} \mu
$$

are boundary values of an analytic function $\tilde{g}(z)$ in $\Omega$. Then the function $h=\left(h_{0}, h_{1}, \ldots, h_{M}\right)$ is given by

$$
h_{j}=\left(\gamma, \phi^{[j]}\right)=\frac{1}{2 \pi} \int_{\Gamma} \gamma(t) \phi^{[j]}(t) \mathrm{d} t,
$$

where $\phi^{[j]}$ are solutions of the following integral equations

$$
\left(\mathbf{I}+\hat{\mathbf{N}}^{*}+\mathbf{J}\right) \phi^{[j]}=-\chi^{[j]} \quad(j=0,1, \ldots, M) .
$$

\section{CIRCULAR SLITS MAP}

Assume that an analytic function $w=f(z)$ maps the boundaries $\Gamma_{j}$, for $j=0,1, \ldots, M$, of $\Omega$ onto the circular slits of $\Omega_{1}$ (Fig. 1). The mapping function $f(z)$ is uniquely determined by assuming that $f(a)=0$ and $f(0)=\infty$, where $0 \in \Omega$ and $a \neq 0$ is a fixed point in $\Omega$ such that the residue of the function $f$ at 0 is equal to $1^{2}$. Hence $f$ can be written in the form

$$
f(z)=\left(\frac{1}{z}-\frac{1}{a}\right) \mathrm{e}^{z g(z)},
$$

where $g$ is an analytic function on $\Omega^{3,4}$.

Note that the boundary values of $f$ can be represented in the form

$$
f\left(z_{j}(t)\right)=\mu_{j} \mathrm{e}^{\mathrm{i} \theta_{j}(t)}, \quad \Gamma_{j}: z=z_{j}(t),
$$

for $j=0,1, \ldots, M$, where $0 \leqslant t \leqslant \beta_{j}, \theta_{j}$ are the boundary correspondence functions of $\Gamma_{j}$, and $\mu_{j}$ are the radii of the circular slits. From (10) and (11) we obtain the following equation

$$
\begin{aligned}
z(t) g(z(t))= & \ln |f(z(t))|-\ln \left|\frac{1}{z(t)}-\frac{1}{a}\right| \\
& -\mathrm{i} \ln \left(\frac{1}{z(t)}-\frac{1}{a}\right)+\mathrm{i} \theta(t) .
\end{aligned}
$$

This equation can be written as

$$
\hat{A}(t) g(z(t))=\gamma(t)+h(t)+\mathrm{i} v(t),
$$

where

$$
\begin{aligned}
& \hat{A}(t)=z(t), \\
& \gamma(t)=-\ln \left|\frac{1}{z(t)}-\frac{1}{a}\right|, \\
& h(t)=\ln \mu(t)=\left(\ln \mu_{0}, \ln \mu_{1}, \ldots, \ln \mu_{M}\right), \\
& \gamma(t)=-\ln \left(\frac{1}{z(t)}-\frac{1}{a}\right)+\theta(t) .
\end{aligned}
$$


By obtaining $h_{0}, h_{1}, \ldots, h_{M}$ from (8), we obtain

$$
\mu_{j}=\mathrm{e}^{h_{j}} \quad \text { for } j=0,1, \ldots, M .
$$

The unit tangent to $\Gamma$ at $z(t)$ is denoted by $T(z(t))=z^{\prime}(t) /\left|z^{\prime}(t)\right|$. It can be shown that ${ }^{9}$

$$
f(z)=\frac{|f(z)|}{\mathrm{i}} T(z) \frac{\left|\theta^{\prime}(t)\right|}{\theta^{\prime}(t)} \frac{f^{\prime}(z)}{\left|f^{\prime}(z)\right|},
$$

and squaring both sides gives

$$
f(z)^{2}=-T(z)^{2}|f(z)|^{2} \frac{f^{\prime}(z)^{2}}{\left|f^{\prime}(z)\right|^{2}} \quad(z \in \Gamma) .
$$

Then the function $D(z)$ defined by

$$
\begin{aligned}
D(z) & =z^{3} f^{\prime}(z) \\
& =z^{3} f(z)\left[z g^{\prime}(z)+g(z)\right]-z \mathrm{e}^{z g(z)}
\end{aligned}
$$

is analytic in $\Omega$.

Combining (14), (13) and (10) we obtain the following boundary relationship

$$
\frac{z^{2} \mathrm{e}^{2 z g(z)}}{a^{2}}=-\left[\frac{\bar{z}|z|}{a-z}|f(z)| T(z) \frac{D(z)}{|D(z)|}\right]^{2},
$$

for $z \in \Gamma$. Comparing (15) and (3) leads us to the choice of $P(z)=D(z), Q(z)=T(z), c(z)=$ $-(z|z||f(z)| / \overline{(a-z)})^{2}, \quad G(z)=z^{2} \mathrm{e}^{2 z g(z)} / a^{2}, \quad$ and $H(z)=0$. Theorem 1 yields

$$
\begin{array}{r}
T(z) D(z)+\frac{1}{2 \pi \mathrm{i}} \int_{\Gamma} K(z, w) T(w) D(w)|\mathrm{d} w| \\
=\frac{\overline{a^{2}} z^{3}|f(z)|^{2}}{(\bar{a}-\bar{z})^{2}} \overline{T(z),}
\end{array}
$$

for $z \in \Gamma$, where

$$
K(z, w)=\frac{(z|z||f(z)|)^{2} \overline{(a-w)^{2} T(z)}}{(w|w||f(w)|)^{2} \overline{(a-z)^{2}(w-z)}}-\frac{T(z)}{w-z} .
$$

Write the preceding integral equation as

$$
F(z)+\int_{\Gamma} N_{T}(z, w) F(w)|\mathrm{d} w|=\frac{\overline{a^{2}} z^{3}|f(z)|^{2}}{(\bar{a}-\bar{z})^{2}} \overline{T(z)},
$$

where

$$
\begin{aligned}
F(z)= & T(z) D(z), \\
D(z)= & z^{3} f^{\prime}(z), \\
N_{T}(z, w)= & \frac{1}{2 \pi \mathrm{i}}\left[\frac{T(z)}{z-w}\right. \\
& \left.-\frac{z|z|^{2}|f(z)|^{2} \overline{(a-w)^{2} T(z)}}{w|w|^{2}|f(w)|^{2} \overline{(a-z)^{2}(z-w)}}\right],
\end{aligned}
$$

are such that

$$
\begin{aligned}
N_{T}(z(t), z(t)) & \\
= & \frac{1}{2 \pi\left|z^{\prime}(t)\right|} \operatorname{Im} \frac{z^{\prime \prime}(t)}{z^{\prime}(t)}-\frac{1}{\pi \mathrm{i}\left|z^{\prime}(t)\right|} \overline{\left[\frac{z^{\prime}(t)}{a-z(t)}\right]} \\
& -\frac{1}{2 \pi \mathrm{i}\left|z^{\prime}(t)\right|} \overline{\left.\frac{z^{\prime}(t)}{z(t)}\right]}-\frac{3}{2 \pi \mathrm{i}\left|z^{\prime}(t)\right|} \frac{z^{\prime}(t)}{z(t)} .
\end{aligned}
$$

Using the single-valuedness of $f$ leads to the following conditions

$$
\frac{1}{2 \pi} \int_{-\Gamma_{j}} \frac{F(w)}{w^{3}}|\mathrm{~d} w|=0 \quad \text { for } j=0,1, \ldots, M .
$$

Applying Cauchy's integral formula we obtain the following conditions

$$
\frac{1}{2 \pi} \int_{\Gamma} \frac{F(w)}{w^{2}}|\mathrm{~d} w|=-\mathrm{i},
$$

and

$$
\frac{1}{2 \pi} \int_{\Gamma} \frac{F(w)}{w}|\mathrm{~d} w|=0 .
$$

Thus the integral equation (16) should give a unique solution provided the parameters $\mu_{j}, j=$ $0,1, \ldots, M$, that appear in $N_{T}(z, w)$ are known. By solving the integral equation (9) we obtain, for $j=$ $0,1, \ldots, M, \phi^{[j]}$, which then gives $h_{j}$ through (8), which in turn gives $\mu_{j}$ through (12). By solving (16) with the known values of $\mu_{j}$ we obtain $F(z)$. Substituting (10) into (13) to get

$$
\left(\frac{1}{z}-\frac{1}{a}\right)^{2} \mathrm{e}^{2 z g(z)}=-T(z)^{2}|f(z)|^{2} \frac{f^{\prime}(z)^{2}}{\left|f^{\prime}(z)\right|^{2}} .
$$

Then taking the complex logarithm gives

$$
g(z)=\frac{1}{2 z} \log \left[\frac{-(a z T(z)|f(z)|)^{2}}{(a-z)^{2}} \frac{f^{\prime}(z)^{2}}{\left|f^{\prime}(z)\right|^{2}}\right],
$$

where $z \in \Gamma$, and the complex logarithm is defined by

$$
\log (z)=\ln |z|+\mathrm{i} \operatorname{Arg}(z),
$$

such that $0 \leqslant \operatorname{Arg}(z) \leqslant 2 \pi$. Finally, the approximate boundary values of $f(z)$ are obtained from (10), i.e.

$$
f(z)=\frac{a-z}{a z} \mathrm{e}^{z g(z)} \quad(z \in \Gamma) .
$$

The approach presented here is an improvement over Sangawi et $\mathrm{al}^{9}$ such that no integral equation for $\theta^{\prime}(t)$ is required here for the computation of $f(z)$. 


\section{PARALLEL SLITS MAP}

Assume a parallel slit region $\Omega_{2}$ in the $w$-plane subtending a given angle $\theta$ with the positive real axis. Assume that an analytic function $w=\hat{f}(z)$ maps $\Omega$ in the z-plane onto $\Omega_{2}$. It is uniquely determined by assuming that $\hat{f}(0)=\infty$ so that the Laurent expansion of $\hat{f}$ near $z=0$ has the form ${ }^{2}$

$$
\hat{f}(z)=\frac{1}{z}+a_{1} z+a_{2} z^{2}+\cdots .
$$

Hence the function $\hat{f}$ can be written in the form

$$
\hat{f}(z)=\frac{1}{z}+\mathrm{e}^{\mathrm{i}(\theta-\pi / 2)} z \hat{g}(z),
$$

where $\hat{g}$ is analytic in $\Omega^{3,4}$. Note that the boundary values of $\hat{f}$ can be represented in the form

$$
\hat{f}\left(z_{p}(t)\right)=\mathrm{e}^{\mathrm{i}(\theta-\pi / 2)}\left[r_{p}+\mathrm{i} S_{p}(t)\right], \Gamma_{p}: z=z_{p}(t),
$$

where $0 \leqslant t \leqslant \beta_{p}$, for $p=0,1, \ldots M$. Let $F(z)=$ $\mathrm{e}^{\bar{A} \hat{f}(z)}=\mathrm{e}^{r_{p}+\mathrm{i} S_{p}(t)}$ with $A=\mathrm{e}^{-\mathrm{i}(\pi / 2-\theta)}$.

From (18) and (19) we obtain the following equation

$$
\begin{aligned}
z(t) \hat{g}(z(t))=r_{p}- & \operatorname{Re}\left(\frac{\bar{A}}{z(t)}\right) \\
& +\mathrm{i}\left(S_{p}(t)-\operatorname{Im}\left(\frac{\bar{A}}{z(t)}\right)\right) .
\end{aligned}
$$

This equation can be written as

$$
\hat{A}(t) \hat{g}(z(t))=\gamma(t)+h(t)+\mathrm{i} v(t),
$$

where

$$
\begin{aligned}
\hat{A}(t) & =z(t), \\
\gamma(t) & =-\operatorname{Re}(\bar{A} / z(t)), \\
h(t) & =r_{p}, \\
v(t) & =S_{p}(t)-\operatorname{Im}(\bar{A} / z(t)) .
\end{aligned}
$$

By using $h_{0}, h_{1}, \ldots, h_{M}$ from (8), we obtain

$$
r_{j}=h_{j} \quad \text { for } j=0,1, \ldots, M \text {. }
$$

Using the unit tangent to $\Gamma$ at $z(t): T(z(t))=$ $z^{\prime}(t) /\left|z^{\prime}(t)\right|$, it can be shown that ${ }^{10}$

$$
F(z)=\frac{|F(z)|}{\mathrm{i}} \frac{\left|S_{p}^{\prime}(t)\right|}{S_{p}^{\prime}(t)} T(z) \frac{F^{\prime}(z)}{\left|F^{\prime}(z)\right|} \quad(z \in \Gamma) .
$$

Define the function $E(z)$ by

$$
\begin{aligned}
E(z) & =z^{3} \mathrm{e}^{-(\bar{A} / z)} F^{\prime}(z) \\
& =z^{3} \mathrm{e}^{z \hat{g}(z)}\left[z \hat{g}^{\prime}(z)+\hat{g}(z)\right]-z \bar{A} \mathrm{e}^{z \hat{g}(z)},
\end{aligned}
$$

which is analytic in $\Omega$.

Combining (24) and squaring both sides of (23) we obtain the following boundary relationship

$$
z^{2} \mathrm{e}^{2 z \hat{g}(z)}=-\frac{\overline{z^{3}}|F(z)|^{2} T(z)^{2}}{z \mathrm{e}^{2 \operatorname{Re}(\bar{A} / z)}} \frac{E(z)^{2}}{|E(z)|^{2}} .
$$

By comparing (25) and (3), we are led to the choice of $P(z)=E(z), \quad Q(z)=T(z), \quad c(z)=$ $-\left(z^{3}|F(z)|^{2}\right) /\left(\bar{z} \mathrm{e}^{2 \operatorname{Re}(\bar{A} / z)}\right), \quad G(z)=z^{2} \mathrm{e}^{2 z \hat{g}(z)}$, and $H(z)=0$. Theorem 1 yields

$$
\begin{aligned}
T(z) E(z)+ & \frac{1}{2 \pi \mathrm{i}} \int_{\Gamma} K(z, w) T(w) E(w)|\mathrm{d} w| \\
& =\frac{z^{3}|F(z)|^{2} A}{\overline{z^{2}} \mathrm{e}^{2 \operatorname{Re}(\bar{A} / z)}} \overline{T(z)} \quad(z \in \Gamma),
\end{aligned}
$$

where $K(z, w)$ is equal to

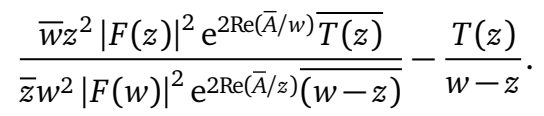

Write the integral equation (26) as

$$
F_{1}(z)+\int_{\Gamma} N_{T}(z, w) F_{1}(w)|\mathrm{d} w|=\frac{z^{3}|F(z)|^{2} A}{\overline{z^{2}} \mathrm{e}^{2 \operatorname{Re}(\bar{A} / z)}} \overline{T(z)},
$$

where $z \in \Gamma$ and

$$
F_{1}(z)=T(z) E(z)
$$

$$
\begin{aligned}
& N_{T}(z, w)=\frac{1}{2 \pi \mathrm{i}}\left[\frac{T(z)}{z-w}\right. \\
& \left.-\frac{\bar{w} z^{3}|F(z)|^{2} \mathrm{e}^{2 \operatorname{Re}(\bar{A} / w)} \overline{T(z)}}{\bar{z} w^{3}|F(w)|^{2} \mathrm{e}^{2 \operatorname{Re}(\bar{A} / z) \overline{(z-w)}}}\right],
\end{aligned}
$$

so that $N_{t}(z(t), z(t))$ is equal to

$$
\begin{aligned}
\frac{1}{2 \pi\left|z^{\prime}(t)\right|} & \left(\operatorname{Im} \frac{z^{\prime \prime}(t)}{z^{\prime}(t)}+\frac{\overline{z^{\prime}(t)}}{\overline{\mathrm{i} z(t)}}\right) \\
& -\frac{3}{2 \pi \mathrm{i}\left|z^{\prime}(t)\right|} \frac{z^{\prime}(t)}{z(t)}-\frac{1}{\pi \mathrm{i}\left|z^{\prime}(t)\right|} \operatorname{Re}\left[\frac{\bar{A} z^{\prime}(t)}{z(t)^{2}}\right] .
\end{aligned}
$$

Using the single-valuedness of the mapping function $\hat{f}$, we are led to the following conditions

$$
\frac{1}{2 \pi \mathrm{i}} \int_{-\Gamma_{j}} \frac{\mathrm{e}^{(\bar{A} / w)} F_{1}(w)}{w^{3}}|\mathrm{~d} w|=0,
$$

for $j=1,2, \ldots, M$. By Cauchy's integral formula, we obtain the following conditions

$$
\frac{1}{2 \pi \mathrm{i}} \int_{\Gamma} \frac{F_{1}(w)}{w^{2}}|\mathrm{~d} w|=-\bar{A},
$$


and

$$
\frac{1}{2 \pi \mathrm{i}} \int_{\Gamma} \frac{F_{1}(w)}{w}|\mathrm{~d} w|=0 .
$$

Thus the integral equation (27) should give a unique solution provided the parameters $|F(z(t))|=\mathrm{e}^{r_{j}(t)}$, $j=0,1, \ldots, M$, that appear in $N_{T}(z, w)$ are known.

By solving the integral equation (9) we obtain $\phi^{[j]}, j=0,1, \ldots, M$, which gives $h_{j}$ through (8) which in turn gives $r_{j}$ through (22). By solving integral equation (27) with the known values of $r_{j}$ we obtain $F_{1}(z)$. From the definition of $F_{1}(z)$, we obtain

$$
F^{\prime}(z)=F_{1}(z) \mathrm{e}^{(\bar{A} / z)} / z^{3} T(z) .
$$

Taking the complex logarithm on both sides of (25) gives the explicit form of $\hat{g}(z)$ as

$$
\hat{g}(z)=\frac{1}{2 z} \log \left[-\mathrm{e}^{(-2 \bar{A}) / z}\left(|F(z)| T(z) \frac{F^{\prime}(z)}{\left|F^{\prime}(z)\right|}\right)^{2}\right],
$$

where the complex logarithm is explained in the previous section. Finally, from the preceding explicit form of $\hat{g}(z)$ and (18), the approximate boundary values of $\hat{f}(z)$ and $\hat{f}^{\prime}(z)$ are given by

$$
\begin{aligned}
\hat{f}(z) & =\frac{1}{z}+A z \hat{g}(z), \quad z \in \Gamma, \\
\hat{f}^{\prime}(z(t)) z^{\prime}(t) & =\frac{A F^{\prime}(z(t)) z^{\prime}(t)}{\mathrm{e}^{(\bar{A} / z(t))+z(t) \hat{g}(z(t))}} \quad(z(t) \in \Gamma) .
\end{aligned}
$$

The approach presented here is an improvement over Sangawi et $\mathrm{al}^{10}$ so that no integral equation for $S^{\prime}(t)$ is required here for the computation of $f(z)$.

\section{INTERIOR AND INVERSE MAPPING FUNCTIONS}

The approximate interior values of the functions $f(z)$ and $\hat{f}(z)$ are calculated by Cauchy's integral formula 9,10

$$
\begin{aligned}
& f(z)=\frac{\frac{a-z}{a z} \int_{\Gamma} \frac{a w f(w)}{a-w} \frac{1}{w-z} \mathrm{~d} w}{\int_{\Gamma} \frac{1}{w-z} \mathrm{~d} w}, \\
& \hat{f}(z)=\frac{\frac{1}{z}+\frac{1}{2 \pi \mathrm{i}} \int_{\Gamma} \frac{\hat{f}(w)-\frac{1}{w}}{w-z} \mathrm{~d} w}{\frac{1}{2 \pi \mathrm{i}} \int_{\Gamma} \frac{1}{w-z} \mathrm{~d} w},
\end{aligned}
$$

where $z \in \Omega$. Numerically, the formulas (34) and (35) have the advantage that the denominators compensate for the error in the numerators ${ }^{16}$. The integrals in (34) and (35) are approximated by the trapezoidal rule.
The inverse mapping function $\tilde{f}^{-1}(w)=z$ are computed by Cauchy's integral formula together with the fact that $\tilde{f}^{-1}(\infty)=0$, i.e.,

$$
z=\tilde{f}^{-1}(w)=\frac{1}{2 \pi \mathrm{i}} \int_{J} \frac{\tilde{f}^{-1}(\zeta)}{\zeta-w} \mathrm{~d} \zeta .
$$

By introducing $\zeta(t)=\tilde{f}(z(t))$, we obtain $z \in \Omega$ by

$$
z=\frac{1}{2 \pi \mathrm{i}} \int_{J} \frac{z(t)}{\tilde{f}(z(t))-w} \tilde{f}^{\prime}(z(t)) z^{\prime}(t) \mathrm{d} t,
$$

where $\tilde{f}=f$ for circular slit region $\Omega_{1}$ and $\tilde{f}=\hat{f}$ for parallel slit region $\Omega_{2}$.

\section{NUMERICAL EXAMPLES}

The trapezoidal rule is the most accurate method for integrating periodic functions numerically ${ }^{17}$. Nyström's method with the trapezoidal rule ${ }^{18}$ is used for solving the above integral equations. The computational details are similar to those in Refs. 3, 4, 13, 14.

For numerical experiments, we have used some test regions of connectivity one, four, eight and fifteen. All the computations were done using MATLAB 7.12.0.635(R2011a). The number of points used in the discretization of each boundary component $\Gamma_{j}$ is $n$. The test regions and their corresponding images are shown in Figs. 2-9.

Example 1 Consider the region $\Omega$ bounded by the unit circle $\Gamma:\left\{z(t)=\mathrm{e}^{\mathrm{i} t}\right\}$,

$$
a=-0.2+0.2 \mathrm{i}, b=0.1-0.6 \mathrm{i}, \theta=\pi / 5 \text {. }
$$

Then the exact mapping functions for circular slit and parallel slit, respectively, are given $b^{2}$

$$
f(z)=\frac{(a-z)}{a z(1-\bar{a} z)}, \mu=\frac{1}{|a|}, \hat{f}(z)=\frac{1}{z}+\mathrm{e}^{2 \mathrm{i} \theta} z,
$$

and $r=0$. Fig. 2 and Fig. 3 show the numerical results based on our method. See Table 1 and Table 2 for the results.

Example 2 Let $\Omega$ be the region bounded by ${ }^{3}$

$$
\begin{aligned}
& \Gamma_{0}:\left\{z(t)=(10+3 \cos 3 t) \mathrm{e}^{\mathrm{i} t}\right\}, \\
& \Gamma_{1}:\left\{z(t)=-3.5+6 \mathrm{i}+0.5 \mathrm{e}^{-\mathrm{i} \pi / 4}\left(\mathrm{e}^{\mathrm{i} t}+4 \mathrm{e}^{-\mathrm{i} t}\right)\right\}, \\
& \Gamma_{2}:\left\{z(t)=5+0.5 \mathrm{e}^{\mathrm{i} \pi / 4}\left(\mathrm{e}^{\mathrm{i} t}+4 \mathrm{e}^{-\mathrm{i} t}\right)\right\}, \\
& \Gamma_{3}:\left\{z(t)=-3.5-6 \mathrm{i}+0.5 \mathrm{e}^{\mathrm{i} \pi / 4}\left(\mathrm{e}^{\mathrm{i} t}+4 \mathrm{e}^{-\mathrm{i} t}\right)\right\},
\end{aligned}
$$

where $0 \leqslant t \leqslant 2 \pi$. We chose $a=8.5+0.1 \mathrm{i}$ and $\theta=\pi / 2$ in circular and parallel slits regions, respectively. Fig. 4 and Fig. 5 show the numerical results based on our method. See Table 3 and Table 4 for the comparison between our computed values of $\mu_{i}$, $r_{i}, i=0, \ldots, 3$, with those computed in Nasser ${ }^{3}$. 

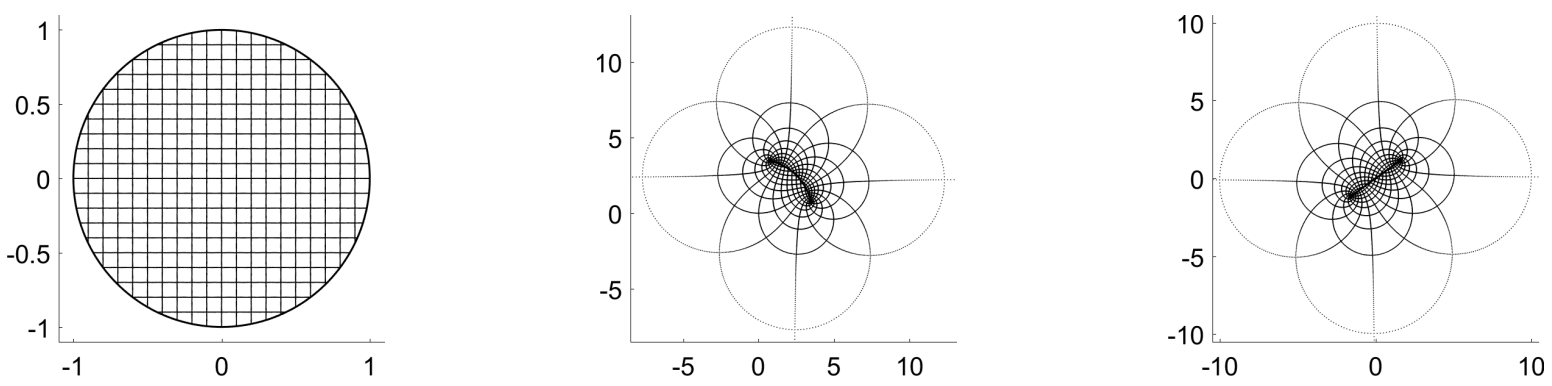

Fig. 2 Mappings a region bounded by unit circle onto canonical regions for Example 1.
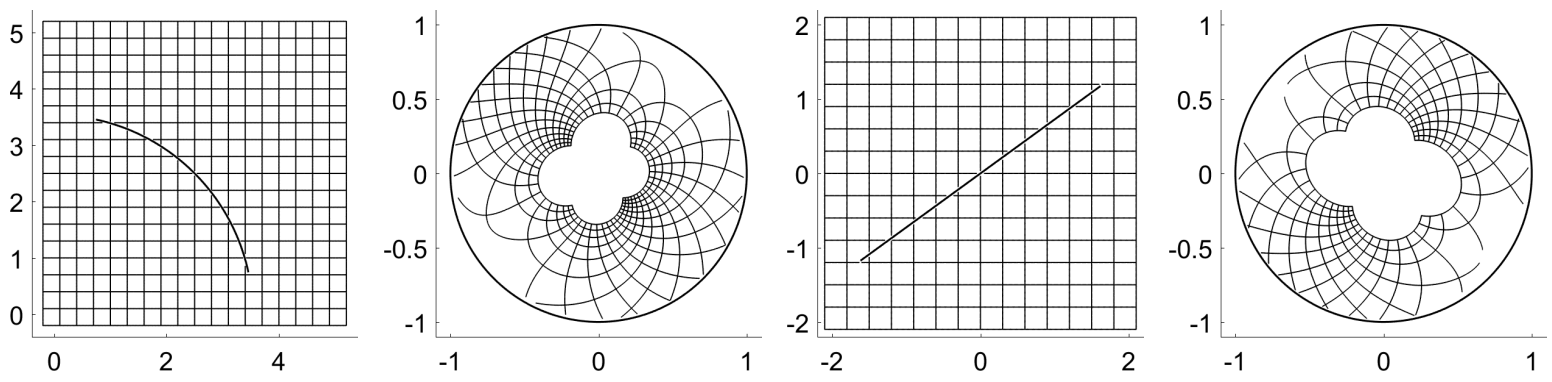

Fig. 3 Inverse images $\left(\Omega_{1}\right.$ and $\Omega_{2}$ to $\Omega$ ) for Example 1 .

Example 3 Consider a region $\Omega$ with complicated boundaries,

$\Gamma_{0}:\left\{z(t)=-0.1-0.4 \mathrm{i}+(6+0.8 \cos 18 t) \mathrm{e}^{\mathrm{i} t}\right\}$

$\Gamma_{1}:\left\{z(t)=2.6-2.6 \mathrm{i}+(1.0+0.6 \cos 4 t) \mathrm{e}^{-\mathrm{i} t}\right\}$,

$\Gamma_{2,3}:\left\{z(t)=\xi_{2,3}+(1.0+0.4 \cos 6 t) \mathrm{e}^{-\mathrm{it}}\right\}$,

$\Gamma_{4,5}:\left\{z(t)=\xi_{4,5}+(1.2+0.4 \cos 8 t) \mathrm{e}^{-\mathrm{i} t}\right\}$,

$$
\begin{aligned}
& \Gamma_{6}:\left\{z(t)=0.1-\mathrm{i}+(0.8+0.6 \cos 2 t) \mathrm{e}^{-\mathrm{i} t}\right\}, \\
& \Gamma_{7}:\left\{z(t)=2.9+1.4 \mathrm{i}+(1.0+0.6 \cos 4 t) \mathrm{e}^{-\mathrm{i} t}\right\},
\end{aligned}
$$

where $0 \leqslant t \leqslant 2 \pi$ and the values of the complex constants are $\xi_{2, \ldots, 5}=(-3.5-1.8 \mathrm{i},-3.3+1.3 \mathrm{i},-0.3+$ $2.7 \mathrm{i},-0.8-3.5 \mathrm{i})$. We chose $a=0.4+1.2 \mathrm{i}$ and $\theta=\pi / 4$ in circular and parallel slits regions, respec-
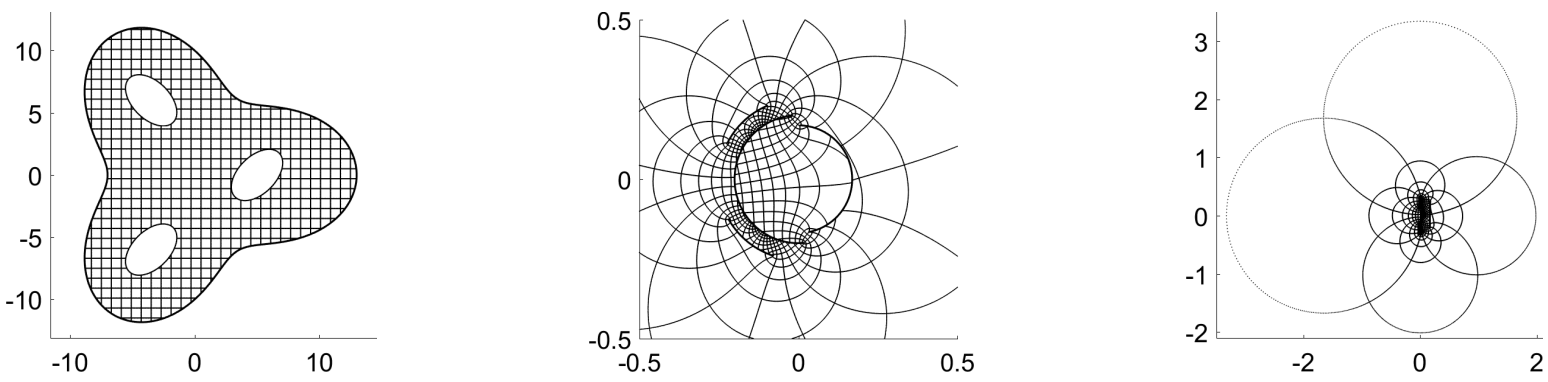

Fig. 4 Mappings of a bounded region of connectivity four onto canonical regions for Example 2.
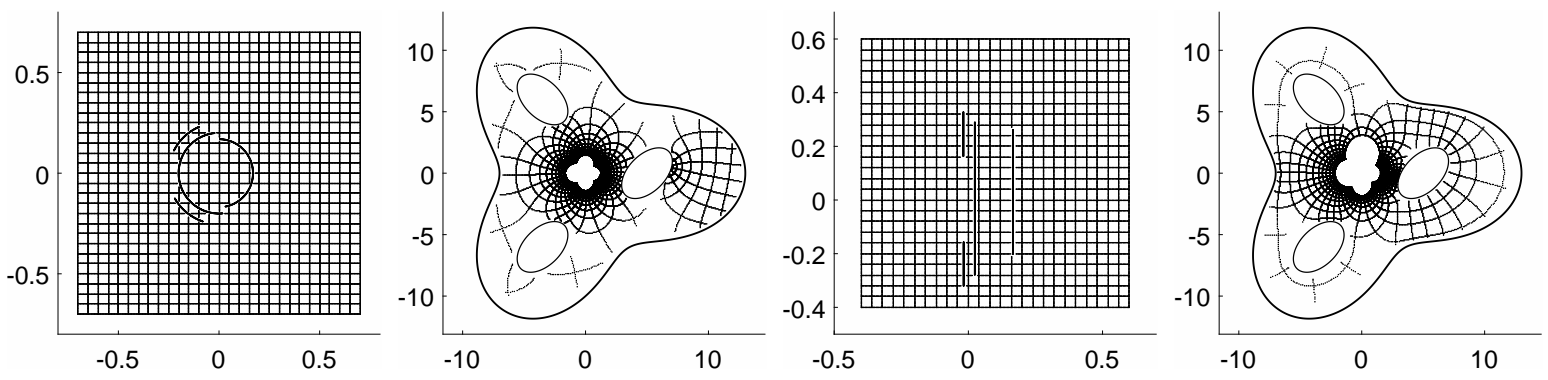

Fig. 5 Inverse images $\left(\Omega_{1}\right.$ and $\Omega_{2}$ to $\Omega$ ) for Example 2 . 

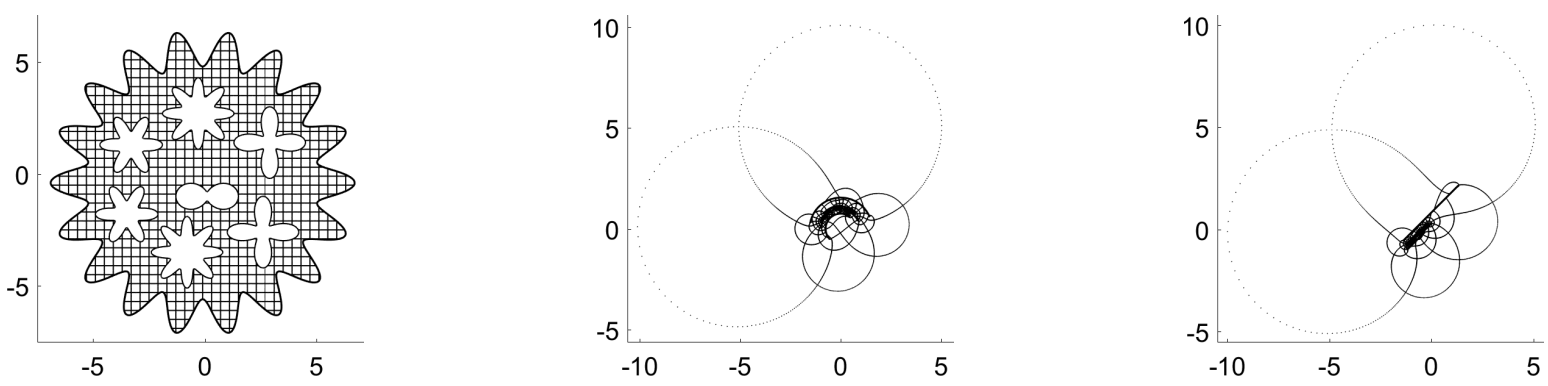

Fig. 6 Mappings of a bounded region of connectivity four onto canonical regions for Example 3.
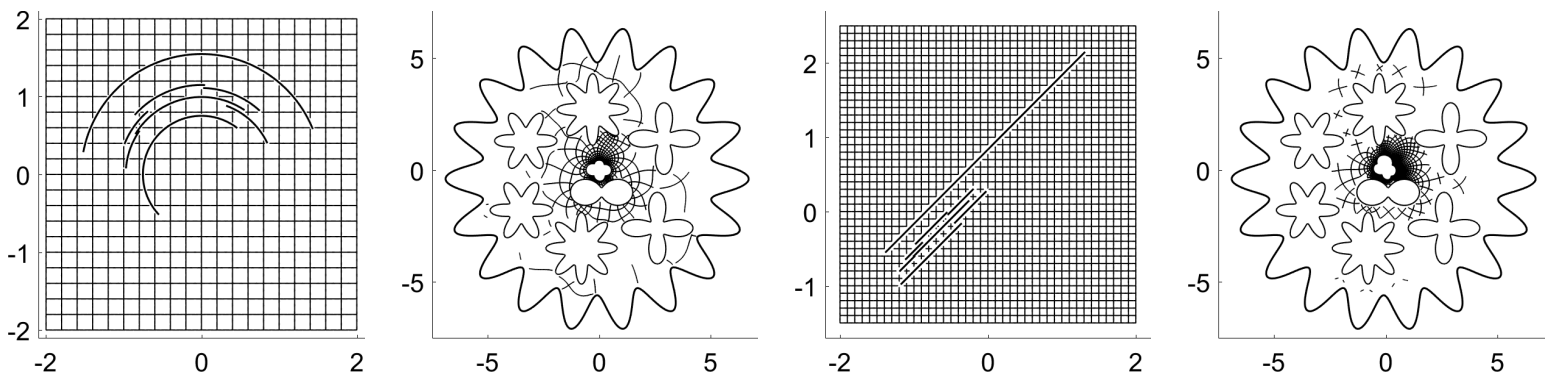

Fig. 7 Inverse images $\left(\Omega_{1}\right.$ and $\Omega_{2}$ to $\Omega$ ) for Example 3 .

Table 1 Error norm for Example 1.

\begin{tabular}{lcc}
\hline$n$ & $\left\|\mu-\mu_{n}\right\|_{\infty}$ & $\left\|f-f_{n}(t)\right\|_{\infty}$ \\
\hline 8 & $1.8 \times 10^{-5}$ & $3.5 \times 10^{-2}$ \\
16 & $3.7 \times 10^{-10}$ & $8.1 \times 10^{-6}$ \\
32 & $8.8 \times 10^{-16}$ & $5.0 \times 10^{-14}$ \\
\hline
\end{tabular}

Table 2 Error norm for Example 1 (unit circle).

\begin{tabular}{ccc}
\hline$n$ & $\left\|r-r_{n}\right\|_{\infty}$ & $\left\|\hat{f}-\hat{f}_{n}(t)\right\|_{\infty}$ \\
\hline 8 & $1.0 \times 10^{-17}$ & $2.3 \times 10^{-2}$ \\
16 & --- & $6.2 \times 10^{-10}$ \\
32 & --- & $1.9 \times 10^{-14}$ \\
\hline
\end{tabular}

tively. Mapping function from the original region onto the circular and parallel slits regions and the inverse mapping functions from the circular and parallel slits regions onto the original region. The numerical results are presented in Fig. 6 and Fig. 7. See Table 5 for our computed values of $\mu_{i}$ and $r_{i}$, $i=0,1, \ldots, 7$.

Example 4 Consider the region of connectivity fifteen with boundaries

$$
z_{j}(t)=\xi_{j}+\mathrm{e}^{\mathrm{i} \sigma_{j}}\left(a_{j} \cos t+\mathrm{i} b_{j} \sin t\right)(j=0, \ldots, 14) .
$$

We chose $a=-2+1.2 \mathrm{i}$ and $\theta=\pi / 2$ in circular and parallel slits regions, respectively. The values
Table 3 Error norm of $\left\|\mu_{j}-\mu_{j n}\right\|_{\infty}$ for Example 2.

\begin{tabular}{lcccc}
\hline$n$ & $j=0$ & $j=1$ & $j=2$ & $j=3$ \\
\hline 64 & $6.8 \times 10^{-13}$ & $1.0 \times 10^{-12}$ & $9.8 \times 10^{-13}$ & $9.7 \times 10^{-13}$ \\
128 & $1.9 \times 10^{-16}$ & $1.0 \times 10^{-15}$ & $2.7 \times 10^{-16}$ & $4.9 \times 10^{-16}$ \\
\hline
\end{tabular}

Table 4 Error norm of $\left\|r_{j}-r_{j n}\right\|_{\infty}$ for Example 2.

\begin{tabular}{lcccc}
\hline$n$ & $j=0$ & $j=1$ & $j=2$ & $j=3$ \\
\hline 64 & $2.7 \times 10^{-12}$ & $2.0 \times 10^{-12}$ & $3.8 \times 10^{-12}$ & $2.0 \times 10^{-12}$ \\
128 & $3.5 \times 10^{-16}$ & $2.0 \times 10^{-16}$ & $5.5 \times 10^{-16}$ & $4.6 \times 10^{-16}$ \\
\hline
\end{tabular}

of the complex constants $\xi_{j}$ and the real constants $a_{j}, b_{j}$, and $\sigma_{j}$ are as in Table 6. The numerical results are presented in Figs. 8-9. See Table 7 for our computed values of $\mu_{i}$ and $r_{i}, i=0,1, \ldots, 14$.

Table 5 The numerical values of $\mu_{i}, r_{i}$, and $\theta_{i}, i=0, \ldots, 7$ for Example 3. $(n=128$. $)$

\begin{tabular}{cccc}
\hline$i$ & $\mu_{i}$ & $r_{i}$ & $\theta_{i}$ \\
\hline 0 & 0.994440701200 & -0.284029138730 & 3.129809929215 \\
1 & 1.110985767458 & -0.353863261497 & 2.292945228462 \\
2 & 1.063686515027 & -0.331000782992 & 2.930879259314 \\
3 & 0.973612244749 & -0.275287566318 & 3.171420702460 \\
4 & 0.753837456220 & -0.139417069130 & 3.636139048989 \\
5 & 1.148572821992 & -0.382534199792 & 2.650117907058 \\
6 & 1.546876218974 & -0.590383326758 & 1.978126115467 \\
7 & 0.938039072007 & -0.208599570836 & 4.573498974480
\end{tabular}



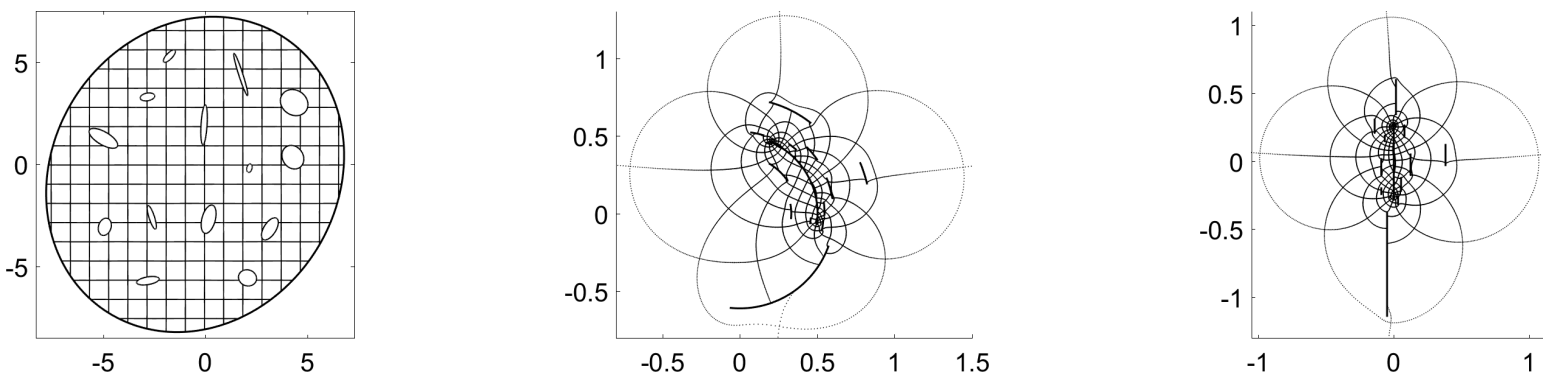

Fig. 8 Mappings of a bounded region of connectivity fifteen onto a canonical regions for Example 4.
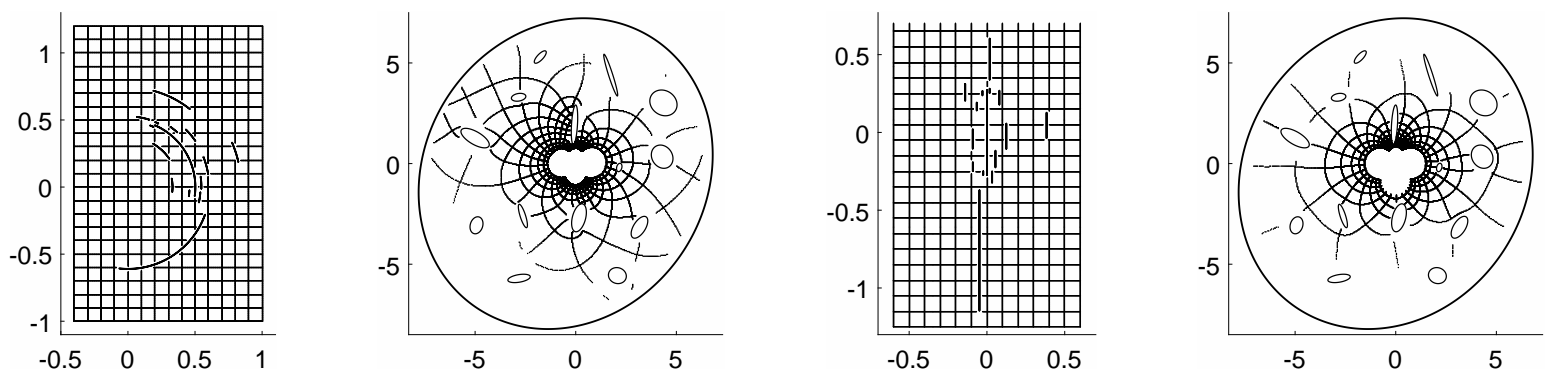

Fig. 9 Inverse images $\left(\Omega_{1}\right.$ and $\Omega_{2}$ to $\Omega$ ) for Example 4 .

Table 6 The values of constants $a_{j}, b_{j}, \xi_{j}$, and $\sigma_{j}$ in Example 4.

\begin{tabular}{lcccc}
\hline$j$ & $a_{j}$ & $b_{j}$ & $\xi_{j}$ & $\sigma_{j}$ \\
\hline 0 & 8.0000 & 7.0000 & $-0.5000-\mathrm{i} 0.5000$ & 1.0000 \\
1 & 0.2976 & -0.6132 & $3.1670-\mathrm{i} 3.1650$ & 5.7197 \\
2 & 0.5061 & -0.6053 & $4.2941+\mathrm{i} 0.3423$ & 0.5778 \\
3 & 0.6051 & -0.7078 & $4.3577+\mathrm{i} 3.0154$ & 4.1087 \\
4 & 0.7928 & -0.3182 & $-5.0000+\mathrm{i} 1.2668$ & 2.6138 \\
5 & 0.3923 & -0.4491 & $2.0694-\mathrm{i} 5.5663$ & 4.4057 \\
6 & 0.3626 & -0.1881 & $-2.8379+\mathrm{i} 3.2940$ & 3.3108 \\
7 & 0.2126 & -0.1281 & $2.1621-\mathrm{i} 0.1940$ & 1.3108 \\
8 & 0.1026 & -1.0881 & $1.7379+\mathrm{i} 4.3960$ & 0.3108 \\
9 & 0.4026 & -0.1481 & $-1.7621+\mathrm{i} 5.2940$ & 0.8108 \\
10 & 0.4323 & -0.3091 & $-4.9306-\mathrm{i} 3.0663$ & 4.4057 \\
11 & 0.5626 & -0.1881 & $-2.8162-\mathrm{i} 5.7060$ & 3.3108 \\
12 & 0.7126 & -0.3281 & $0.1621-\mathrm{i} 2.6940$ & 1.3108 \\
13 & 0.1343 & -0.6088 & $-2.6262-\mathrm{i} 2.6040$ & 0.3108 \\
14 & 0.9826 & -0.1481 & $-0.0621+\mathrm{i} 1.9294$ & 1.5108 \\
\hline
\end{tabular}

\section{GRAPHICAL USER INTERFACE}

In this section, we presented a graphical user interface (GUI) to illustrate our finding. The graphical user interface was created by using MATLAB. More numerical results including graphical and image transformation can be computed by using GUI. There are two modes in the GUI which is graph mode or image mode. To have a graphical result, we choose the graph mode and set the input values for the numbers of nodes and the parameters for
Table 7 The numerical values of $\mu_{i}, r_{i}$, and $\theta_{i}, i=$ $0, \ldots, 14$ for Example 4. $(n=128)$

\begin{tabular}{cccc}
\hline$i$ & $\mu_{i}$ & $r_{i}$ & $\theta_{i}$ \\
\hline 0 & 0.5023222796 & 0.0008589109 & 0.3587111366 \\
1 & 0.6086785849 & 0.0799766244 & 0.4382073558 \\
2 & 0.6055849127 & 0.1247855487 & 0.2768221012 \\
3 & 0.5474464053 & 0.0547912084 & 0.2466426059 \\
4 & 0.3734163069 & -0.0912331525 & 0.5310740652 \\
5 & 0.5491862962 & 0.0172876271 & 0.4385971607 \\
6 & 0.3324241335 & -0.0907118361 & 0.1035010586 \\
7 & 0.8438900618 & 0.3832088812 & 0.2438605063 \\
8 & 0.5410588262 & 0.0332667860 & 0.0627077746 \\
9 & 0.4591054253 & -0.0237558527 & 0.1653659457 \\
10 & 0.4873520274 & -0.0656146300 & 0.5919062426 \\
11 & 0.5249755441 & -0.0287033882 & 0.5158620847 \\
12 & 0.7420010973 & 0.0175966334 & 0.7842061900 \\
13 & 0.5267875577 & -0.1397473330 & 0.8848907532 \\
14 & 0.6102168256 & -0.0481344748 & -0.8175689388 \\
\hline
\end{tabular}

the boundaries. There are three kinds of boundary available in the GUI which are circles, ellipses and star shape. The interior points are then selected after the boundaries had been selected. There are two modes of mapping available, which include circular slits and parallel slits. The transformations are computed based on clicking a point $a$ inside the region. For image transformation, we choose the image mode and load the image with selected 


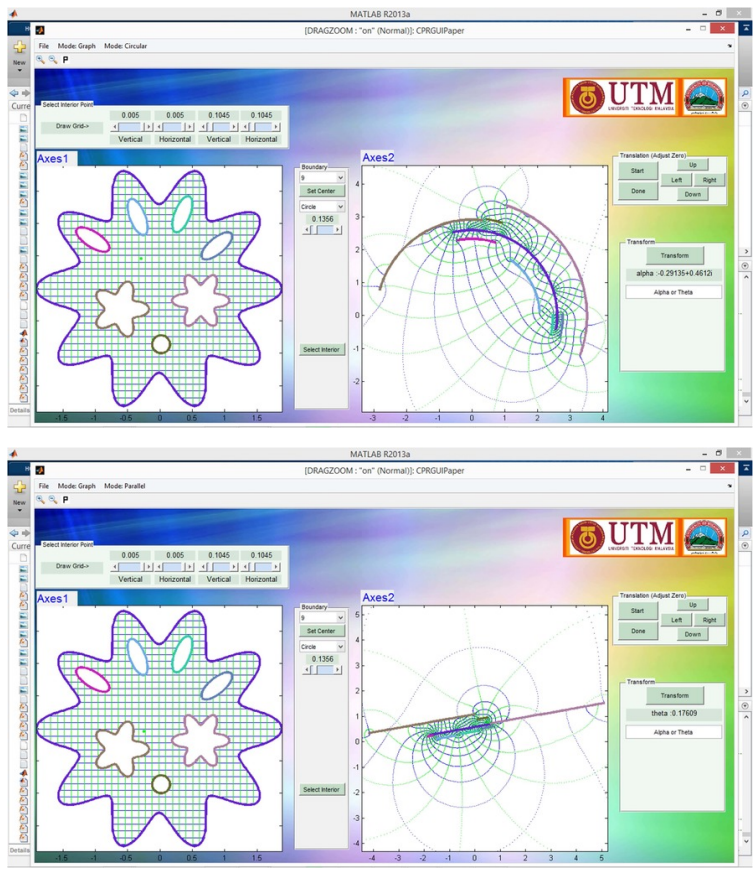

Fig. 10 GUI graphical presentation based circular slits map and parallel slits map.

size. The image can be cropped based on the boundaries selected on the image or the growcut algorithm ${ }^{19}$. The parameterization of the cropped image by growcut algorithm is based on parametric cubic spline, which approximate two real functions $x(t)$ and $y(t)$ using periodic cubic spline and the parameterization $z(t)$ is defined as ${ }^{20,21}$

$$
z(t)=x(t)+\mathrm{i} y(t) \quad(0 \leqslant t \leqslant 2 \pi) .
$$

The computation of the transformation is similar as the graph mode. The quality of the transformed image improves with increasing number of nodes.

Fig. 10 presents a GUI about slits mapping. The boundary options in GUI are circle, ellipse and star shapes. Fig. 11 presents image transformation results from GUI for image cropped based on selected boundaries. Fig. 12 presents image transformation results from GUI for image cropped based on growcut algorithm.

\section{CONCLUSIONS}

In this paper, we have constructed new boundary integral equations for conformal mapping of bounded multiply connected regions onto a circular slit region and parallel slit region. The advantage of our method is that our boundary integral equations are all linear and continuous. Several mappings of

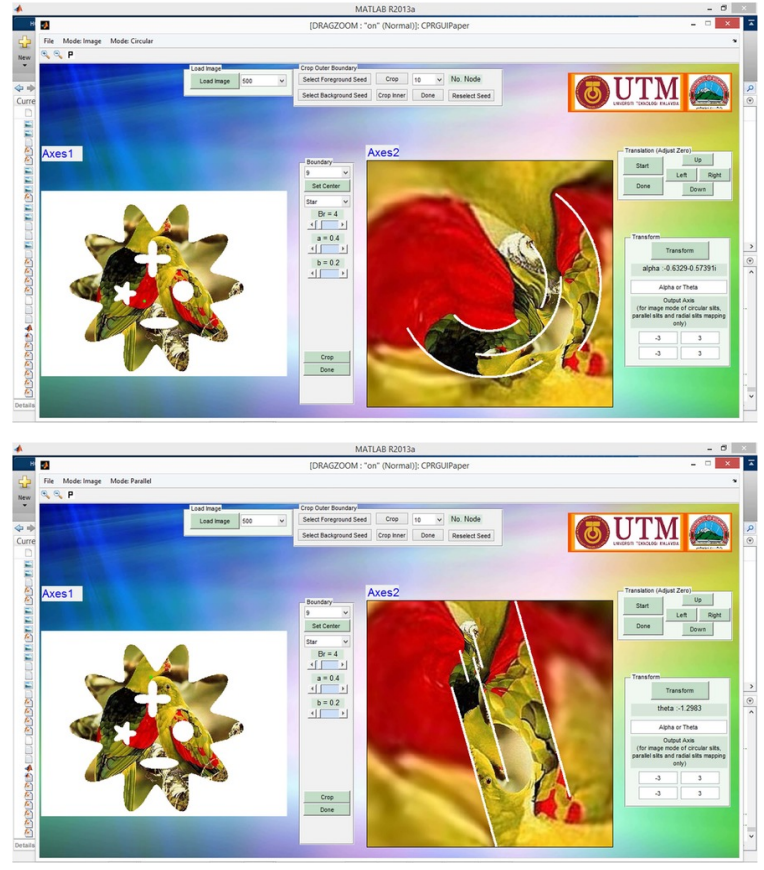

Fig. 11 GUI image processing presentation based circular slits map and parallel slits map.

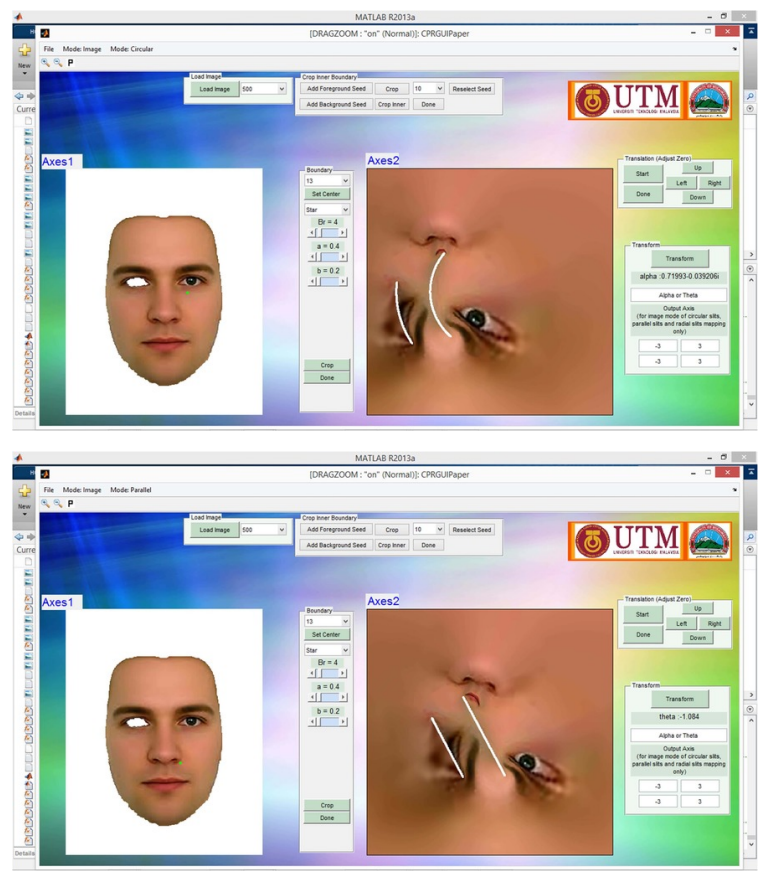

Fig. 12 GUI image processing with cropped face based circular slits map and parallel slits map.

the test regions of connectivity one, four and fifteen were computed numerically using the proposed method. After the boundary values of the mapping 
function are computed, the interior values of the mapping function and its inverse are calculated by means of Cauchy integral formula. The numerical examples presented for the three canonical regions have illustrated that our boundary integral equation method has high accuracy.

Acknowledgements: This work was supported in part by the Malaysian Ministry of Education through the Research Management Centre, Universiti Teknologi Malaysia, UTM-CIAM (Vote: R.J130000.7809.4F637) and the Kurdistan Ministry of Higher Education through Department of Mathematics, School of Science, University of Sulaimani. These supports are gratefully acknowledged. The authors would like to thank all the referees for valuable comments and suggestions.

\section{REFERENCES}

1. Wen GC (1992) Conformal Mapping and Boundary Value Problems, English translation of Chinese edition 1984, American Mathematical Society.

2. Nehari Z (1952) Conformal Mapping, Dover, New York.

3. Nasser MMS (2009) A boundary integral equation for conformal mapping of bounded multiply connected regions. Comput Meth Funct Theor 9, 127-43.

4. Nasser MMS (2009) Numerical conformal mapping via boundary integral equation with the generalized Neumann kernel. SIAM J Sci Comput 31, 1695-715.

5. Nasser MMS (2011) Numerical conformal mapping of multiply connected regions onto the second, third and fourth categories of Koebe's canonical slit domains. J Math Anal Appl 382, 47-56.

6. Wegmann R, Nasser MMS (2008) The RiemannHilbert problem and the generalized Neumann kernel on multiply connected regions. $J$ Comput Appl Math 214, 36-57.

7. Amano K (1994) A charge simulation method for the numerical conformal mapping of interior, exterior and doubly connected domains. J Comput Appl Math 53, 350-70.

8. DeLillo TK, Driscoll TA, Elcrat AR, Pfaltzgraff JA (2008) Radial and circular slit maps of unbounded multiply connected circle domains. Proc Roy Soc A 464, 1719-37.

9. Sangawi AWK, Murid AHM, Nasser MMS (2012) Circular slits map of bounded multiply connected regions. Abstr Appl Anal 389, 1-26.

10. Sangawi AWK, Murid AHM, Nasser MMS (2012) Parallel slits map of bounded multiply connected regions. J Math Anal Appl 389, 1280-90.

11. Sangawi AWK, Murid AHM, Nasser MMS (2011) Linear integral equations for conformal mapping of bounded multiply connected regions onto a disk with circular slits. Appl Math Comput 218, 2055-68.
12. Sangawi AWK, Murid AHM, Nasser MMS (2012) Annulus with circular slit map of bounded multiply connected regions via integral equation method. Bull Malays Math Sci Soc 35, 945-59.

13. Murid AHM, Hu LN (2009) Numerical experiment on conformal mapping of doubly connected regions onto a disk with a slit. Int $J$ Pure Appl Math 51, 589-608.

14. Murid AHM, Hu LN (2009) Numerical conformal mapping of bounded multiply connected regions by an integral equation method. Int J Contemp Math Sci 4, 1121-47.

15. Nasser MMS, Murid AHM, Ismail M, Alejaily EMA (2011) Boundary integral equation with the generalized Neumann kernel for Laplace's equation in multiply connected regions. Appl Math Comput 217, 4710-27.

16. Halsing J, Ojala R (2008) On the evaluation of layer potentials close to their sources. J Comput Phys 227, 2899-921.

17. Davis PJ, Rabinowitz P (1984) Methods of Numerical Integration, 2nd edn, Academic Press, Orlando.

18. Atkinson KE (1997) The Numerical Solution of Integral Equations of the Second Kind, Cambridge Univ Press, Cambridge.

19. Vezhnevets V, Konouchine V (2005) GrowCutInteractive multi-label N-D image segmentation by cellular automata. In: Proceedings of the 15th International Conference on Computer Graphics and Applications GraphicCon'2005, pp 150-6.

20. Graham NY (1983) Smoothing with periodic cubic splines. Bell Syst Tech J 62, 101-10.

21. Faires JD, Burden R (2003) Numerical Methods, 3rd edn, Thomson-Brooks/Cole, Pacific Grove, CA. 